\title{
Linx
}

Revue des linguistes de l'université Paris X Nanterre

5 | 1994

La négation

\section{Les phrases négatives elliptiques}

\section{David Gaatone}

\section{(2) OpenEdition \\ Journals}

Édition électronique

URL : http://journals.openedition.org/linx/1201

DOI : 10.4000/linx.1201

ISSN : 2118-9692

\section{Éditeur}

Presses universitaires de Paris Nanterre

\section{Édition imprimée}

Date de publication : 1 juin 1994

Pagination : 163-175

ISSN : 0246-8743

\section{Référence électronique}

David Gaatone, "Les phrases négatives elliptiques », Linx [En ligne], 5 | 1994, mis en ligne le 18 juillet 2012, consulté le 19 avril 2019. URL : http://journals.openedition.org/linx/1201 ; DOI : 10.4000/ linx.1201

Ce document a été généré automatiquement le 19 avril 2019.

Département de Sciences du langage, Université Paris Ouest 


\title{
Les phrases négatives elliptiques
}

\author{
David Gaatone
}

\section{NOTE DE L'AUTEUR}

Ces quelques réflexions doivent beaucoup aux notes personnelles sur les constructions averbales que Claire Blanche-Benveniste a bien voulu me communiquer.

1 Rien que de très normal sans doute à ce qu'un colloque sur le non se tienne sur les lieux mêmes où éclatèrent, il y a un quart de siècle, des incidents issus du refus, du rejet, d'un certain système, termes intimement liés comme chacun sait à la négation polémique ou à la négation tout court. Pas de surprise non plus dans le fait qu'un colloque sur le non ait lieu peu de temps après la victoire difficile du oui. Enfin, nulle bizarrerie dans la formulation des trois premières phrases, y compris celle-ci, de mon entrée en matière. Elle illustre, comme on n'aura pas manqué de le remarquer, le titre de cette très brève étude, titre qu'il serait sans doute préférable de modifier légèrement, en remplaçant «elliptiques », terme couvrant dans la littérature trop de phénomènes différents, par " averbales", plus précis.Les trois phrases d'ouverture sont donc des phrases négatives averbales, dans ce sens que le prédicat y est représenté, plutôt que par un verbe, par un terme appartenant à l'inventaire classique de la négation syntaxique. Bien sûr, j'aurais pu présenter les choses d'une façon plus positive, en remplaçant ces termes négatifs, là du moins où c'est possible, par leurs correspondants positifs. Mais on voit immédiatement que cela m'aurait amené à utiliser une syntaxe plus "ordinaire ", c'est-à-dire, avec un verbe, comme, par exemple, dans le cas de la première : il y a quelque chose de très normal à ce que.... C'est précisément là que réside l'essentiel de mon propos : attirer l'attention sur l'existence, en tant que phénomène productif de phrases averbales, où l'omission du verbe est conditionnée, plus exactement rendue possible, par la présence d'une négation. J'insisterai d'abord sur le fait qu'il ne s'agit pas de ce qu'un linguiste tel que Ch. Bally (1965:159) appelait effectivement l'ellipse, contextuelle ou situationnelle, c'est-à-dire, la non-répétition en discours d'une information récupérable, soit dans le contexte linguistique, auquel cas on a affaire en réalité à un substitut de forme zéro, comme dans 
1:

1. Ce pays compte beaucoup de politiciens, mais pas d'hommes d'Etat soit dans les circonstances de l'énonciation comme dans 2 :

2. Surtout pas ça!

2 Il ne s'agit pas non plus de diverses constructions syntaxiques, souvent exclamatives, où l'absence d'un verbe est non seulement admise, mais même quelquefois de rigueur. Ainsi, dans 3-8, la phrase averbale existe parallèlement à la verbale :

3. (C'est) un petit génie (que) cet étudiant!

4. (Il est) excellent, ce café ! (Il n'est) pas fameux, ce café !

5. Autant (il y a) d'hôtels, autant (il y a) de bruits de clefs

6. (C'est) impossible ! *Possible! Merveilleux !

7. Quoi (qu'y a-t-il) de plus rabâché que ces slogans!

8. Vous n'avez pas assez osé, de là (vient ?) votre échec

Dans 9, en revanche, seule la phrase averbale est possible :

9. Quelle calamité que ce personnage!

3 Les phrases négatives averbales qui nous intéressent n'impliquent aucune information verbale donnée ailleurs, donc aucun " effacement », et ne sont pas liées à des structures syntaxiques particulières, telles que celles illustrées par les exemples ci-dessus, dont certaines d'ailleurs n'admettent pas de négation $(5,9)$. Elle pourraient être rangées sous ce que Bally appelle la « sous-entente » (ibid.), terme plutôt malheureux, qui signifie chez lui, la possibilité, pour un signe, de fonctionner, sans réalisation explicite dans un contexte donné, par association avec un autre contexte linguistique de même valeur, où il figure explicitement : travailler la nuit/pendant la nuit, je viens vous demander/pour vous demander.

4 Les phrases négatives averbales peuvent se présenter sous diverses formes, résumées par les formules suivantes :

10. DET.NEG. (nul, aucun, plus aucun, pas un, plus un) NOM

a. Nul bruit de vie que le chant des grenouilles

b. Aucun silence en cet homme, aucun mystère

c. Plus aucun bruit de tam-tam

d. Pas une phrase de ce livre où il ne se soit donné tout entier

e. Le Bien m'a rincé l'âme : plus une goutte de venin

11. ADV.NEG. (pas, plus, jamais, guère, peu) DE NOM

a. Pas de cheveux du tout chez cet Hercule-là !

b. Plus de satisfaction, soudain, dans ses yeux ni sur ses lèvres

c. Jamais de mesquinerie, de jalousie, de malice

d. Guère de journée où je ne m'interroge sur elles

e. Peu de genres plus conventionnels que le film américain

5 On constatera, au passage, que là encore, peu de, dont l'orientation négative et, donc, la proximité avec la négation, ont été souvent relevées, a un comportement similaire à celui des négations proprement dites, et cela, bien entendu, contrairement à un peu de.

12. ADV.NEG. (pas, plus, jamais) SUPERLATIF (ou équivalent) NOM

a. C'est beau, non? Pas le plus petit signe d'acquiescement.

b. Ces bâtiments bordent le trottoir sur 400 mètres; pas la moindre fenêtre, pas même une lucarne

c. Jamais la moindre explication

d. Pas l'ombre d'un Poulidor, pas trace d'un Bahamontès 
13. PRO.NEG. (personne, rien)

a. Mais ici personne pour les voir

b. Personne dans la cour, quand ses phares balayèrent l'esplanade

c. Rien à opposer aux grandes œuvres du XVIème siècle

d. Dans tout cela, rien qui rappelle la motivation des mots

Il faut encore mentionner d'autres types de phrases négatives averbales, dont les exemples paraissent relativement nombreux dans les textes, mais qui semblent devoir être écartés du fait de leur caractère figé. Le plus important sans doute répond à la formule suivante :

14. ADV.NEG. (pas, plus, ?guère) ADJ. DE INF./QUE $P$

a. Pas étonnant qu'il ait accepté de venir/ ?*Etonnant qu'il ait accepté de venir

b. Plus possible d'éluder la question/*(Encore) possible d'éluder la question

c. Pas facile de survivre dans un tel pays/ ?*Facile de survivre dans un tel pays

Une autre structure, très proche de la précédente, comporte un adjectif à préfixe négatif :

15. (IN-ADJ.) DE INF./ QUE P

a. Impossible de les déloger de leurs certitudes/*Possible de les déloger de leurs certitudes

b. Inutile de fouiller à droite ni à gauche/*Utile de fouiller à droite et à gauche

8 Malgré leur fréquence, ces exemples sont en fait limités à quelques rares adjectifs et ne peuvent donc pas être considérés comme représentant un schéma productif. Les adjectifs à valeur de vérité, ainsi que les adjectifs appréciatifs, que l'on rencontre couramment en position d'attribut dans les constructions impersonnelles, ne sont pas admis comme introducteurs de phrases négatives averbales, pas plus d'ailleurs qu'ils n'autorisent des phrases positives averbales :

16a. Il n'est pas clair que ça change/*Pas clair que ça change/*Clair que ça change

b. Il n'est pas naturel de réagir ainsi/*Pas naturel de réagir ainsi/*Naturel de réagir ainsi

c. Il n'est pas vrai que tout soit perdu/*Pas vrai que tout soit perdu/*Vrai que tout est perdu

d. Il n'est pas douteux que ça change/*Pas douteux que ça change/*Douteux que ça change

Notons que, à $16 \mathrm{~d}$, on peut opposer 16e, où un sens équivalent trouve son expression dans une phrase négative averbale de type productif :

16e. Nul/aucun doute que ça change

D'autre part, certains adjectifs non négatifs autorisent des phrases averbales :

17a. Qu'est-ce que ces gens pensent? Difficile de se l'imaginer

b. Avec Robert Houdin, difficile de dire où finit le rêve

11 La plupart des adjectifs négatifs à préfixe in ne donnent pas de meilleurs résultats :

18a. Il est inexact que cela soit dû au hasard/*Inexact que cela soit dû au hasard

b. Il est illogique que personne n'y ait pensé/*Illogique que personne n'y ait pensé

c. Il est idiot d'accuser la météo du mauvais temps/*Idiot d'accuser la météo du mauvais temps

On conclura qu'il ne suffit pas d'une négation préfixale ou syntaxique pour obtenir une phrase averbale naturelle. Les exemples donnés ci-dessus sont limités à quelques unités lexicales spécifiques. Cependant, le phénomène n'est pas restreint à un petit nombre 
d'adjectifs. Il s'étend également à plusieurs expressions impersonnelles, figées soit sous la forme positive, soit sous la forme négative, soit sous les deux :

19a. Il (n')est(pas/plus)besoin de- *Besoin de- Pas/plus besoin de

b. Il (n')y a(pas/plus)moyen de -*moyen de - Pas/plus moyen de

c. Ce n'est pas la peine de/que - Pas la peine de/que

d. Il n'y a pas/plus de doute que -Pas/plus de doute que

e. Il (n')est (pas/plus) question de/que - *Questions de/que - Pas/plus question de/que

13 Revenons-en à nos structures averbales productives. Toutes sont centrées autour d'un nom, plus précisément un nominal. Leur temps, en l'absence d'un verbe, est, comme pour toutes les phrases averbales, le présent, ou tout autre temps imposé par un éventuel contexte. Comme tous les énoncés averbaux également, elles ne sont pas possibles en subordination:

20a. Je crois qu'il n'y a rien d'étrange à cela/*Je crois que rien d'étrange à cela b. On voit qu'il n'y a pas d'issue/*On voit que pas d'issue

14 Il faut cependant noter la possibilité de subordonnées averbales avec des adverbes de négation ou d'affirmation tels que non et oui :

21. Je crois que non/que oui

L'interprétation des négatives averbales consiste toujours dans l'expression d'une quantité nulle, de l'inexistence, d'une absence. Il y a serait approprié dans tous les exemples (R. Martin 1966 :115-116). En revanche, les expressions avec être, telles que il est ou c'est, des phrases impersonnelles, mais non il est, équivalent littéraire de il y a, ne sont pas omissibles dans ces mêmes contextes, bien que leur charge sémantique soit pratiquement nulle. C'est là un fait digne d'attention, dans la mesure où être, verbe copule, paraît effaçable dans de nombreux contextes, sans donner lieu à des différences de sens perceptibles, comme on a pu le vérifier sur les exemples 4 et 6 , et comme le montrent les exemples suivants :

22a. Je crois (qu'il est) facile de réussir

b. Ces problèmes semblent (être) insolubles

c. Le ministre qu'on savait (être) à Paris...

d. Une fois (que) le chat (est) parti, les souris dansent

e. Bien que (il fût) fatigué, Jean reprit le travail

On voit donc que les cas d'ellipse du verbe être avec des adjectifs (14 et 15), dont on a constaté qu'ils n'étaient pas productifs, ne sont pas de la même espèce que les schémas productifs. Autrement dit, la négation d'une propriété n'autorise pas, du moins d'une façon systématique, l'absence du verbe, comme le fait celle de la quantité, de l'existence. Et comme on peut s'y attendre dans le cas de phrases existencielles, les prédicats nominaux des phrases négatives averbales ont des déterminants indéfinis, sauf, bien entendu, là où l'article défini est exigé par le superlatif (structure 12, pas la moindre réaction), ou dans certaines expressions figées (pas la peine de). On notera que toute autre séquence de type ADV.NEG. DET.(défini)N suppose un contexte de substitution, et donc ne répond pas à notre critère de phrase négative averbale :

23a. - Le client s'est enfui ? - Pas le client, le vendeur

b. Il n'y a pas la guerre là-bas, au moins/*Pas la guerre là-bas au moins

c. Pas de guerre là-bas, au moins

17 Les phrases négatives averbales rendent problématique la formulation traditionnelle de certains phénomènes liés à la négation. Il s'agit essentiellement des adverbes de négation, 
que leur évolution a rapprochés des quantifieurs non négatifs. Dans ces phrases, en effet, pas de, plus de, de même que beaucoup de, trop de, etc... sont uniquement des modifieurs du nom suivant, et non du verbe ou du syntagme verbal tout entier (cf. aussi C. Muller 1987 :669). C'est peut-être ce qui explique l'absence des adverbes négatifs aucunement et nullement de ces constructions, comme le montrent les exemples suivants :

24a. Pas de/nulle/aucune trace de peur dans son regard b. *Aucunement/nullement de trace de peur dans son regard

18 Ces adverbes, en effet, comme l'avait déjà noté Clédat (1901:110), portent plutôt sur le verbe que sur le nom et ne désignent pas la quantité nulle d'un objet. En outre, l'occurrence de de négatif ne peut plus être formulée en termes de fonction du syntagme nominal suivant, ni d'ailleurs de sa position (objet direct ou sujet postposé), puisque ce SN est le centre même de la phrase et ne se trouve donc pas dans la dépendance d'un verbe. Rappelons qu'il existe encore un autre cas où de apparaît en l'absence d'un verbe, celui où il se trouve derrière sans, mais séparé de lui par un élément quelconque (D. Gaatone 1992 :95-96) :

25. Sans presque d'exceptions/ Sans, je crois, d'exceptions

La place de l'adverbe négatif ne peut pas non plus être formulée par rapport au verbe : il ne suit pas le verbe, comme il est dit en général dans la règle, mais précède le nom. Mais, parallèlement ces adverbes négatifs diffèrent des quantifieurs positifs, en ce sens qu'ils impliquent, par eux-mêmes, l'affirmation d'inexistence, ou la négation d'existence, ce qui leur permet de fonctionner systématiquement sans l'aide de ce prédicat verbal d'existence qu'est il y $a$.

Il y a là, je crois, une piste intéressante à explorer, tant du point de vue de la description des phrases averbales, domaine encore assez mal connu, que de celui de la négation.

\section{BIBLIOGRAPHIE}

Bally, Ch., 1965, Linguistique générale et Linguistique française. Ed. Francke, Berne

Clédat, L., 1901, « La préposition et l'article partitif », Revue de Philologie française et de Littérature, XV, pp. 81-131.

Gaatone, D., 1992, « De négatif entre la syntaxe et la sémantique. Réflexions sur quelques propriétés du déterminant de », Langue Française, 94, pp. 93-102.

Martin, R., 1966, Le mot rien et ses concurrents en français. Paris, Klincksieck.

Muller, Cl., 1987, La négation en français. Syntaxe, sémantique et éléments de comparaison avec les autres langues romanes. Thèse de doctorat d'Etat, Université de Paris-7. 


\section{RÉSUMÉS}

La possibilité d'une phrase elliptique ou, plus précisément, d'une phrase averbale, est, en français, soit soumise à des contraintes contextuelles comme, par exemple, la non-répétition d'une information déjà donnée, soit limitée à quelques rares structures syntaxiques, telles que, par exemple, divers types de phrases exclamatives. On constate cependant une certaine affinité entre la présence d'une négation formelle et l'ellipse du verbe. En fait, la négation semble pouvoir fonctionner, en association avec le terme nié, comme prédicat de phrase, rendant ainsi superflue, mais non impossible, la présence d'un support verbal, à condition qu'il s'agisse d'une négation de quantité, d'existence. On tente de décrire les types productifs de phrases négatives averbales, qui doivent être soigneusement distingués des types figés, fréquents dans le discours, mais limités à quelques items lexicaux. L'existence du phénomène mène en outre à revoir la formulation de certaines règles de négation, traditionnellement centrées sur le verbe.

Elliptic, or rather, verbless sentences, are, as a rule, submitted in French to contextual constraints, such as, for instance, erasing of previously given information, or limited to few syntactic structures, such as, for instance, some kinds of exclamative sentences. However, a certain affinity can be found in French between formal negation and verb ellipsis. in fact, a negative word seems to be able to function, together with the negated element, as a predicate, making thereby the verb superfluous, though not forbidden, if the meaning is negation of quantity, of existence. The various productive types of those negative verbless sentences are described, and also carefully distinguished from frozen types, very frequent in speech, but limited to few lexical items. The taking into account of such a phenomenon should lead to new formulations of some rules of negation, traditionnally centered around the verb.

\section{AUTEUR}

\section{DAVID GAATONE}

Université de Tel-Aviv 